\title{
Tumour microenvironment of both early- and late-stage colorectal cancer is equally immunosuppressive
}

\author{
A O'Toole ${ }^{1,2}$, A J Michielsen ${ }^{1,3}$, B Nolan ${ }^{1}$, M Tosetto ${ }^{1}$, K Sheahan ${ }^{1,2}$, H E Mulcahy ${ }^{1,2}$, D C Winter ${ }^{1,2}$, \\ J M Hyland ${ }^{1,2}$, P R O'Connell ${ }^{1,2}$, D Fennelly ${ }^{1,2}$, D O'Donoghue ${ }^{1,2}$, J O'Sullivan ${ }^{3}$, G A Doherty ${ }^{1,2}$ \\ and E J Ryan ${ }^{\star}, 1,2$
}

${ }^{1}$ Centre for Colorectal Disease, St Vincent's University Hospital, Dublin 4, Ireland; ${ }^{2}$ School of Medicine and Medical Sciences, University College Dublin, Dublin 4, Ireland and ${ }^{3}$ Department of Surgery, Institute of Molecular Medicine, Trinity Centre for Health Sciences, St James's Hospital, Dublin 8, Ireland

Background Tumour microenvironment (TME) of advanced colorectal cancer (CRC) suppresses dendritic cell (DC) maturation. Here, our aim was to determine how the microenvironment of early-stage tumours influences DCs.

Methods: Tumour-conditioned media (TCM) was generated by culturing explant tumour tissue in vitro $(n=50)$. Monocyte-derived DCs (MDDCs) of healthy donors or cancer patients were pretreated with TCM and stimulated with lipopolysaccharide (LPS). DC maturation was assessed by flow cytometry and cytokine production measured by ELISA.

Results: TCM from both early- and late-staged tumours abrogated LPS-induction of IL-12p70 secretion, while increasing IL-10. The profile of inflammatory mediators in TCM was similar across stages, and all increased pSTAT3 expression by DCs. CRC patient DCs $(n=31)$ secreted low levels of IL-12p70 and failed to upregulate expression of maturation markers in response to LPS. Furthermore, in vitro culture of autologous DCs with TCM did not change the hypo-responsiveness of patient DCs.

Conclusion: Our data demonstrates that the TME of all stages of CRC contains inflammatory mediators capable of suppressing local DCs. MDDCs obtained from CRC patients are hyporesponsive to stimuli such as LPS. Measures to reverse the negative influence of the TME on DCs will optimise cancer vaccines in both early- and late-stage CRC.

Chronic inflammation in the tumour microenvironment (TME) is integral to the neoplastic process; enhancing tumour growth, invasion, angiogenesis and metastasis (Lin and Karin, 2007). Dendritic cells (DCs) are a key player in orchestrating the immune response in the TME (Palucka and Banchereau, 2012). We have previously demonstrated that the TME of advanced colorectal cancer (CRC) suppresses monocyte-derived dendritic cell (MDDC) maturation and IL-12p70 secretion (Michielsen et al, 2011). Importantly, the degree of this inhibition correlated with the survival of stage IV patients receiving bevacizumab therapy (Michielsen et al, 2012). Although changes in DC phenotype have been noted in both colorectal (Gulubova et al, 2012) and ovarian
(Scarlett et al, 2012) cancer progression, it is not clear if the immunosuppressive nature of the TME varies with disease progression in colorectal cancer.

In a p53-dependant model of ovarian carcinoma depletion of DC in early stages of disease accelerated tumour expansion; but at later stages of disease significantly delayed malignant progression (Scarlett et al, 2012). This suggests a bi-modal role for DCs in cancer progression. However, it is not known if or when a switch in the microenvironmental regulation of DC function occurs in CRC progression (Seton-Rogers, 2012). A clearer understanding of how the suppressive nature of the tumour microenvironment influences the function of DCs in different cancer patient cohorts would 
facilitate development of more effective immunotherapy and DCbased cancer vaccines.

Here we used our established tumour explant model (Michielsen et al, 2011, 2012) to determine if the microenvironment of earlier staged tumours is as suppressive as that of patients with metastatic disease. In addition we characterised the phenotype of non-metastatic CRC patients' MDDCs and examined production of IL-12p70 in response to lipopolysaccharide (LPS) in both the absence and presence of tumour-conditioned media.

\section{MATERIALS AND METHODS}

Ex vivo tumour explant culture. Surgically resected tumour tissue was collected from patients with CRC $(n=50)$. Patient characteristics and details of tumour pathology are presented in Table 1. Sixty four percent of the cohort was male. Median age at diagnosis was 67.4 years (range, 32-93). Tumour-conditioned media (TCM) was prepared as previously described (Michielsen et al, 2011). Briefly, the explanted tissue was cut into four equal-sized pieces of approximately $5 \mathrm{~mm}^{3}$ and cultured (in 24-well plates) in $2 \mathrm{ml}$ RPMI 1640 containing $100 \mathrm{U} \mathrm{ml}^{-1}$ penicillin, $100 \mu \mathrm{g} \mathrm{ml}^{-1}$ streptomycin, $4 \mu \mathrm{g} \mathrm{ml}^{-1}$ fungizone, $30 \mu \mathrm{g} \mathrm{ml}^{-1}$ gentamicin (Invitrogen; Carlsbad, CA, USA) and supplemented with $20 \%$ fetal bovine serum (Invitrogen). After $72 \mathrm{~h}$ in culture, TCM was collected and stored at $-20^{\circ} \mathrm{C}$ until used for analyses. The protocol was approved by the Ethics Committee of St. Vincent's University Hospital.

Monocyte-derived dendritic Cells. Peripheral blood mononuclear cells (PBMCs) were obtained from buffy coat preparations (National Blood Centre, St James's Hospital, Dublin) and from healthy control or patient samples by density gradient centrifugation (Lymphoprep, Axis Shield, Dundee, UK). CD14 ${ }^{+}$monocytes were obtained by positive selection using anti-CD14 microbeads as described by the manufacturer (Miltenyi Biotec, Bergisch Gladbach, Germany). Immature DCs were generated from CD14 ${ }^{+}$ monocytes as described (Michielsen et al, 2011). On day 6 , immature DCs were harvested. Immature MDDCs were treated with a $1: 2$ dilution of TCM from tumour explants for $4 \mathrm{~h}$ before adding $1 \mu \mathrm{g} \mathrm{ml}^{-1}$ of Escherichia coli lipopolysaccharide (LPS; Alexis Biochemicals, Lausen, Switzerland). Cultures were incubated for a further $18 \mathrm{~h}$ at $5 \% \mathrm{CO}_{2}$ and $37^{\circ} \mathrm{C}$.

Analysis of DC maturation. Gating on $\mathrm{CD}_{11 c^{+}}$cells, the expression of DC maturation markers was determined by flow cytometry using the following antibodies: CD11c (B-ly6), CD83 (HB15e), CD80 (L307.4); CD54 (HA58), CCR7 (3D12), CD86

Table 1. Characteristics of the cohort of colorectal cancer (CRC) patients and summary of the pathology of the tumours used to generate tumourconditioned media $(n=50)$

\begin{tabular}{|l|c|c|c|c|}
\hline & Stage I & Stage II & Stage III & Stage IV \\
\hline $\mathrm{N}$ & 8 & 21 & 14 & 7 \\
\hline Male/Female & $6 / 2$ & $15 / 6$ & $9 / 5$ & $2 / 5$ \\
\hline Age, median (range) & $66(46-80)$ & $73(53-84)$ & $65(32-93)$ & $66(58-82)$ \\
\hline CRC mortality & 1 & 4 & 1 & 4 \\
\hline $\begin{array}{l}\text { Lymphovascular } \\
\text { invasion }\end{array}$ & 0 & 9 & 11 & 6 \\
\hline Perineural infiltration & 0 & 0 & 4 & 2 \\
\hline Mismatch repair loss & 1 & 2 & 3 & 0 \\
\hline KRAS mutation & Not tested & Not tested & 4 & 6 \\
\hline Tumour budding & 1 & 0 & 3 & 3 \\
\hline
\end{tabular}

(IT2.2), HLA-DR (TU36) (all from BD Biosciences, Oxford, UK). Cells were also stained with the corresponding isotype control. Samples were acquired on a FACScalibur flow cytometer and data were analysed using Flowjo Software (Tree Star Inc., Ashland, OR, USA).

Cytokine analysis. Cytokines were quantified by ELISA using the manufacturer's protocol; IL-10, IL-12p70 (R\&D Systems, Oxford, UK) and IL-6 (Biolegend, San Diego, CA, USA). The detection limits of the assays were as follows; IL-10, $31.2 \mathrm{pg} \mathrm{ml}^{-1}$; IL-12p70, $31.2 \mathrm{pg} \mathrm{ml}^{-1}$ and IL-6, $7.8 \mathrm{pg} \mathrm{ml}^{-1}$.

TCM from all stages of CRC was screened for the following cytokines: Angiogenin, EGF, ENA-78, bFGF, GRO, IFN- $\gamma$, IGF-1, IL-6, IL-8, Leptin, MCP-1, PDGF-BB, PIGF, RANTES, TGF $\beta 1$, TIMP1, Thrombopoietin, VEGF and VEGF-D using a Cytokine Antibody Array (RayBiotech, Inc, Norcross, GA, USA) according to the manufacturer's protocol.

Phospho-STAT3 analysis. Immature MDDCs $\left(1 \times 10^{6}\right.$ cells per $\mathrm{ml}$ ) were incubated at $37^{\circ} \mathrm{C}, 5 \% \mathrm{CO}_{2}$ for $2 \mathrm{~h}$, and then treated with either recombinant IL-6 (R\&D Systems) at $20 \mathrm{ng} \mathrm{ml}^{-1}$ or a $1: 2$ dilution of TCM for $15 \mathrm{~min}$. Cells were fixed with Cytofix buffer (BD Biosciences), permeabilized with Phosflow Perm Buffer III (BD Biosciences) and pSTAT3 expression was measured using phospho-STAT3 (BD Biosciences) following the recommended protocol. Samples were acquired on a FACScalibur flow cytometer.

Statistical analysis. Statistical analyses were performed using GraphPad Prism version 5.00 for Windows (GraphPad software, La Jolla, CA, USA). ANOVA with the Kruskal-Wallis post hoc test was used to compare multiple groups of nonparametric data. The Wilcoxon signed-rank test was used to compare two groups of paired nonparametric data, whereas the Mann-Whitney $U$-test to compare two groups of unpaired data. $P$-values $<0.05$ were considered significant in all the analyses.

\section{RESULTS}

TME of all stages of colorectal cancer modulates MDDC response to LPS. Tumour-conditioned media (TCM) was generated from CRC tumours with different stages of disease progression (stage I, $n=8$; stage II, $n=21$; stage III, $n=14$, stage IV, $n=7$ ). Tumours were assigned as stage I-IV according to the American Joint Committee on Cancer (AJCC) classification system (Edge and Compton, 2010). We found that TCM from all stages of CRC significantly suppressed IL-12p70, whereas levels of IL-10 were increased in response to LPS (Figure 1A). TCM pretreatment of MDDCs also suppressed the upregulation of maturation markers in response to LPS (Figure $1 \mathrm{~B}$ and $\mathrm{C}$ ). Immature MDDCs were cultured with TCM without the addition of LPS as a control, however this had no effect on cytokine secretion or maturation marker expression when compared with immature DCs cultured in media alone (data not shown). These data clearly illustrate that the microenvironment of both early and advanced colorectal cancer has the potential to potently suppress cell-mediated immunity.

Profile of inflammatory mediators in both the early and advanced colorectal cancer microenvironment. TCM generated from CRC stages I-IV were screened for the presence of a panel of inflammatory mediators using an antibody based protein-array. Figure 2A shows that TCM of all stages of CRC progression contains a similar profile of inflammatory mediators. Of note, high levels of IL-6, IL-8, GRO and angiogenin are present. Using ELISA we confirmed that IL- 6 was present in equivalent concentration in TCM from all tumour stages (Figure 2B).

Next we determined if TCM of colorectal tumours equally induce STAT3 phosphorylation in DCs. Briefly, MDDCs were incubated for $15 \mathrm{~min}$ with a $1: 2$ dilution of TCM from stage I-IV 

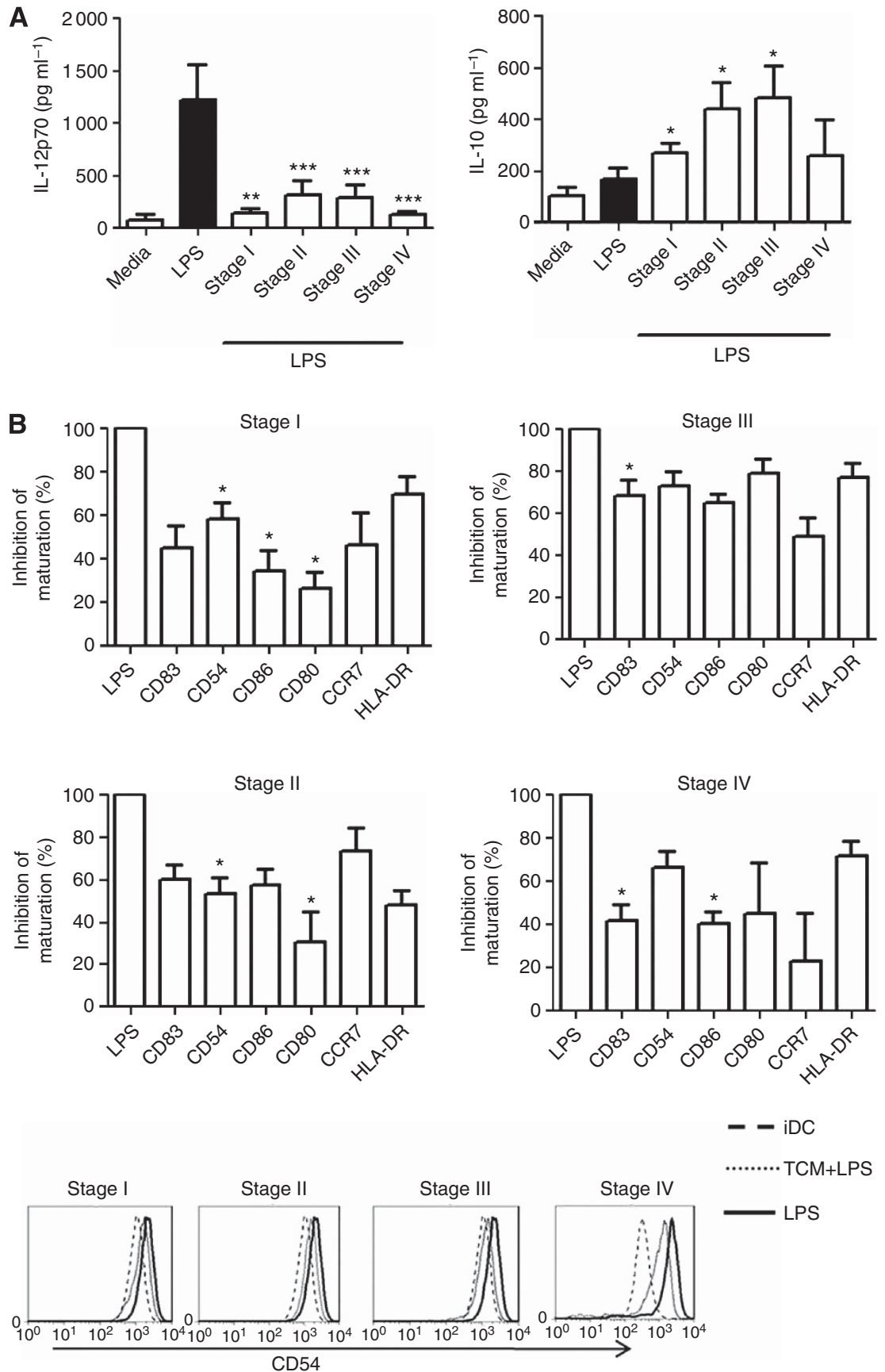

Figure 1. TME of all colorectal cancer stages suppresses DC maturation. Immature monocyte-derived DCs were treated for $4 \mathrm{~h}$ with tumourconditioned media (TCM). LPS $\left(1 \mu \mathrm{g} \mathrm{ml}^{-1}\right)$ was added and the cells cultured for a further $18 \mathrm{~h}$. Dendritic cells were cultured in media alone as a negative control and LPS stimulated dendritic cells with no TCM pretreatment were included as a positive control. (A) Supernatants were collected and the levels of IL-12p70 and IL-10 were measured by ELISA. Error bars presented are s.e.m. Statistical differences were determined by ANOVA. ${ }^{\star} P<0.05,{ }^{*} P<0.01$ and ${ }^{\star \star *} P<0.001$. (B) Expression of DC maturation markers were assessed by flow cytometry. Percentage inhibition was calculated relative to cells stimulated with LPS alone. Representative flow cytometry histograms from one experiment showing CD54 expression are shown. Statistical significance was calculated using the Wilcoxon signed-rank test. ${ }^{\star} P<0.05$.

CRC ( $n=5$, per stage) or recombinant IL-6 (positive control), and levels of pSTAT3 measured by flow cytometry. We found that four of the five patients' TCM of each stage I-III induced significant upregulation of pSTAT3 expression by MDDCs, however TCM from five of the five cases tested with stage IV disease TCM induced pSTAT3 expression (Figure 2C).

MDDCs of stage II colorectal cancer patients are hypo-responsive to LPS. Next, to determine if the local microenvironment of the tumour could modulate the function of autologous DCs, we cultured MDDC from presurgery blood samples in the presence of TCM generated from the patient's own tumour $(n=13$, stage I, $n=1$, stage II, $n=11$, stage III, $n=1)$. Median age of the cohort was 60.8 years (range, 50-84). Nine patients were male. Further clinical details of this cohort are presented in Table 2. TCM used in these experiments suppressed the responsiveness of healthy control MDDCs to LPS as we have previously observed (Figure 3). However, as the response of the patient MDDCs to LPS was poor, TCM pretreatment of patient DC did not have an additional inhibitory effect. Of particular note, MDDCs obtained from this 
A

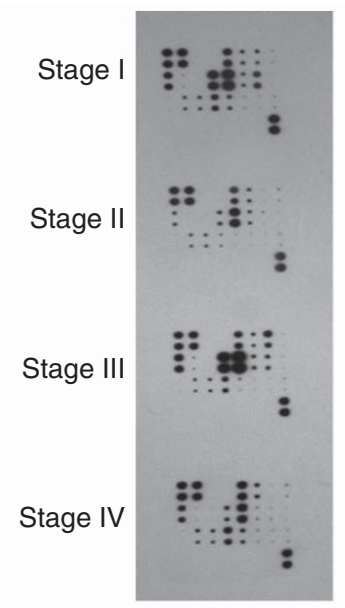

C

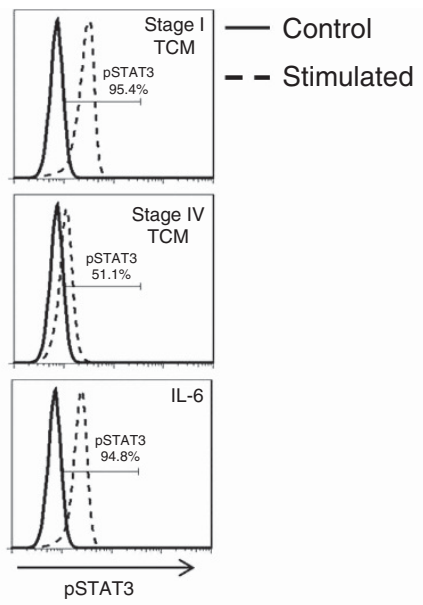

\begin{tabular}{|c|c|c|c|c|c|c|c|}
\hline POS & POS & NEG & NEG & Angiogenin & EGF & ENA78 & bFGF \\
POS & POS & NEG & NEG & Angiogenin & EGF & ENA78 & bFGF \\
\hline GRO & IFN- $\gamma$ & IGF-1 & IL-6 & IL-8 & Leptin & MCP-1 & PDGF-BB \\
GRO & IFN- $\gamma$ & IGF-1 & IL-6 & IL-8 & Leptin & MCP-1 & PDGF-BB \\
\hline PIGF & RANTES & TGF- $\beta 1$ & TIMP1 & TIMP2 & Thrombopoietin & VEGF & VEGF-D \\
PIGF & RANTES & TGF- $\beta 1$ & TIMP1 & TIMP2 & Thrombopoietin & VEGF & VEGF-D \\
\hline BLANK & BLANK & BLANK & BLANK & BLANK & BLANK & NEG & POS \\
\hline BLANK & BLANK & BLANK & BLANK & BLANK & BLANK & NEG & POS \\
\hline
\end{tabular}

B

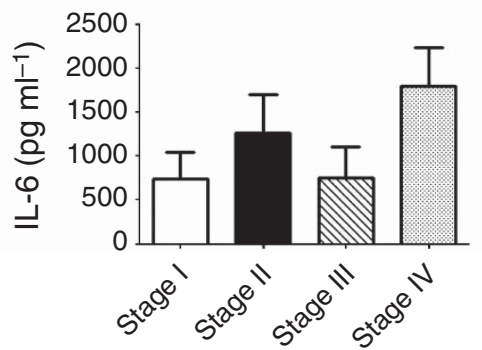

Figure 2. TME of all colorectal cancer stages contains high levels of inflammatory mediators including IL-6. TCM of all stages of colorectal cancer was screened for the presence of a number of inflammatory mediators using an antibody array. High levels of IL-6, IL-8, GRO, angiogenin and TIMP1 were expressed in TCM of all stages (A). Levels of IL-6 were quantified by ELISA in TCM from all stages of CRC ( $n=5$ per stage) (B). DCs isolated from healthy controls were stimulated for $15 \mathrm{~min}$ with IL-6 $\left(100 \mathrm{ng} \mathrm{ml}^{-1}\right)$ or cultured with a $1: 2$ dilution of TCM of stage I or stage IV TCM. The levels of PSTAT3 were analysed by flow cytometry (C).

cohort of patients with early-staged CRC secreted very low levels of IL-12p70 in response to LPS. Although pretreatment of patient or control MDDCs with TCM increased IL-10 production in response to LPS, this did not reach statistical significance in these experiments.

In addition, MDDCs were obtained from a further $n=31$ patients with CRC before surgery. Clinical and pathological details of this cohort are presented in Table 2. (stage I, $n=6$; stage II, $n=15$; stage III, $n=9$; stage IV, $n=1$, with a median age at diagnosis 63 years (44-82)). Interestingly, we found that in general the patients' MDDCs secreted low levels of IL-12p70 in response to LPS (median, $39.8 \mathrm{pg} \mathrm{ml}^{-1}$, (6.9-990 $\left.\mathrm{pg} \mathrm{ml}^{-1}\right)$.

\section{DISCUSSION}

Tumour-derived factors drive the evolution of an immunosuppressive network, thus contributing to tumour invasiveness and metastasis (Kim et al, 2006; Gabrilovich et al, 2012). A deeper understanding of this process in humans is urgently needed to optimise immunotherapeutic strategies. In this study, using tumour tissue explants we confirm that the TME of all stages of colorectal cancer exerts a significant suppressive effect on DCs.

Previous work has suggested that colorectal cancer patients have both numerical and functional impairment in peripheral blood (Della Porta et al, 2005) and local tumour-infiltrating DCs (Schwaab et al, 2001; Dadabayev et al, 2004; Inoue et al, 2005; Cui et al, 2007; Yuan et al, 2008). We found that MDDCs obtained from CRC patients with non-metastatic disease were hyporesponsive to LPS, secreting markedly reduced levels of IL-12p70 compared with MDDCs obtained from healthy controls. Decreased $I L-12 B$ transcription in monocytes obtained from patients with CRC was previously reported to be associated with the stage of CRC (Stanilov et al, 2012). Yet, in our study, MDDCs of those patients with non-metastatic disease (stage I and II) were just as nonresponsive to LPS as those with metastatic disease. Data from a recently reported clinical study indicate the absolute requirement 
Table 2. Characteristics of the cohort of patients with CRC used to characterise phenotype of MDDCs (A) and the characteristics of

patients with CRC used to investigate the effect of TCM on autologous MDDCs (B)

\section{\begin{tabular}{l|l|l|l|l} 
Stage I & Stage II & Stage III & Stage IV
\end{tabular}}

(A) MDDCs obtained from patients with colorectal cancer $(n=31)$

\begin{tabular}{|l|c|c|c|c}
\hline N & 6 & 15 & 9 & 1 \\
Male/Female & $4 / 2$ & $9 / 6$ & $3 / 6$ & Male \\
Lymphovasular & 0 & 5 & 5 & 1 \\
invasion & & & & \\
Perineural infiltration & 0 & 1 & 3 & 1 \\
Mismatch repair loss & 0 & 1 & 2 & 0 \\
KRAS mutation & Not tested & Not tested & 0 & 1 \\
Tumour budding & 1 & 0 & 3 & 3
\end{tabular}

(B) Autologous TCM and MDDCs obtained from patients with colorectal cancer $(n=13)$

\begin{tabular}{|c|c|c|c|}
\hline $\mathrm{N}$ & 1 & 11 & 1 \\
\hline Male/Female & Male & $8 / 3$ & Female \\
\hline Age (median, range) & 63 & $68.5(50-84)$ & 51 \\
\hline $\begin{array}{l}\text { Lymphovascular } \\
\text { invasion }\end{array}$ & 0 & 1 & 1 \\
\hline Perineural infiltration & 0 & 1 & 0 \\
\hline Mismatch repair loss & 0 & 1 & 0 \\
\hline KRAS mutation & Not tested & Not tested & 0 \\
\hline Tumour budding & 1 & 1 & 0 \\
\hline
\end{tabular}

for IL-12p70 secretion for the efficacy of DC therapies (Carreno et al, 2013), highlighting the need to understand how IL-12p70 is regulated in the TME. Our finding that the TME of all CRC tumours tested $(n=50)$ potently suppressed IL-12p70 secretion may also help explain why often DC vaccines that are capable of inducing T-cell responses in vitro may fail in vivo (Rossowska et al, 2009).

Local activation of NF- $\mathrm{KB}$ and resultant inflammatory cytokines are of great importance in the shaping of the local tumour environment (Muthuswamy et al, 2012). We screened the TME of both metastatic and non-metastatic tumours to determine if there were different patterns of local inflammation and found high levels of inflammatory mediators, notably IL-6 and IL-8, in all the samples. IL- 8 can contribute to colon carcinogenesis by increasing local infiltration and retention of immature myeloid cells (Feijoo et al, 2005; Asfaha et al, 2013). IL-6 also promotes accumulation of myeloid derived suppressor cells (MDSCs; Bunt et al, 2007). In addition, IL-6-mediates arginase activation and the subsequent reduction in MHC class II expression on DC, a mechanism critical for inducing dysfunction of the immune system in the tumourbearing state (Narita et al, 2013). The activation of STAT3dependent pathways (downstream of IL-6, among others) may be critical in mediating the influence of the TME on DC differentiation and function (Nefedova et al, 2004). We found TME of both metastatic and non-metastatic tumours upregulated pSTAT3 expression in MDDCs. Interestingly, STAT3-depleted human DCs with adenoviral STAT3 short hairpin RNA were capable of producing more cytokines with TLR stimulation and were resistant to cancer-derived factors, inducing tumour Ag-specific $\mathrm{T}$ cells more efficiently than control DCs (Iwata-Kajihara et al, 2011).

To conclude we have demonstrated that MDDCs of nonmetastatic CRC patients secrete low levels of IL-12p70 in response to LPS and that the TME of CRC of all stages of disease progression is able to suppress the function of MDDCs. These data add some strength to the argument in favour of some form of
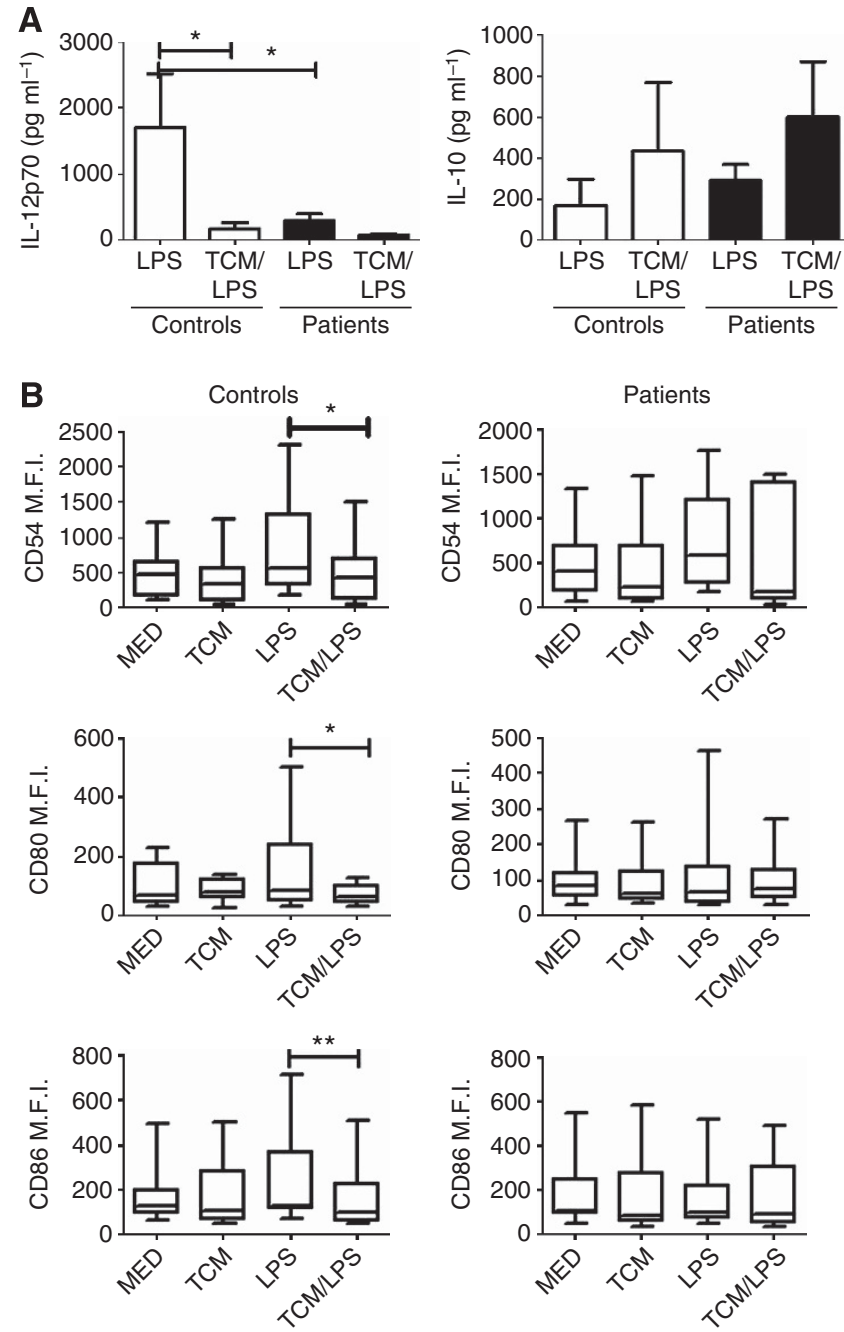

Figure 3. MDDCs obtained from patients with early-staged CRC respond poorly to LPS, secreting low levels of IL-12p70. Peripheral blood monocytes were obtained from colorectal cancer patients before surgical removal of their tumour. Tumour explants of these patients were also obtained, cultured in vitro for $72 \mathrm{~h}$ and TCM collected. (stage $I, n=1$, stage $I I, n=11$, stage $I I, n=1)$. MDDCs were pretreated for $4 \mathrm{~h}$ with TCM and then LPS $\left(1 \mu \mathrm{g} \mathrm{ml}^{-1}\right)$ added and cells cultured for a further $18 \mathrm{~h}$. Levels of IL-12p70 and IL-10 in supernatants were determined by ELISA (A). Expression of maturation markers (CD54, CD80 and CD86) were measured by flow cytometry (B). Statistical differences were determined using Wilcoxon signed-rank test. ${ }^{\star} P<0.05,{ }^{* \star} P<0.01$.

preoperative immune boosting strategy to increase disease-free survival following surgical resection (Lonnroth et al, 2008).

\section{ACKNOWLEDGEMENTS}

Aoibhlinn O'Toole was funded by a Newman Fellowship from the University College Dublin Foundation, sponsored by AbbVie.

\section{REFERENCES}

Asfaha S, Dubeykovskiy AN, Tomita H, Yang X, Stokes S, Shibata W, Friedman RA, Ariyama H, Dubeykovskaya ZA, Muthupalani S, Ericksen R, Frucht H, Fox JG, Wang TC (2013) Mice that express human interleukin- 8 have increased mobilization of immature myeloid cells, 
which exacerbates inflammation and accelerates colon carcinogenesis. Gastroenterology 144: 155-166.

Bunt SK, Yang L, Sinha P, Clements VK, Leips J, Ostrand-Rosenberg S (2007) Reduced inflammation in the tumor microenvironment delays the accumulation of myeloid-derived suppressor cells and limits tumor progression. Cancer Res 67: 10019-10026.

Carreno BM, Becker-Hapak M, Huang A, Chan M, Alyasiry A, Lie WR, Aft RL, Cornelius LA, Trinkaus KM, Linette GP (2013) IL-12p70-producing patient DC vaccine elicits Tc1-polarized immunity. J Clin Invest 123: 3383-3394.

Cui G, Yuan A, Goll R, Olsen T, Husebekk A, Vonen B, Florholmen J (2007) Distinct changes of dendritic cell number and IL-12 mRNA level in adjacent mucosa throughout the colorectal adenoma-carcinoma sequence. Cancer Immunol Immunother 56: 1993-2001.

Dadabayev AR, Sandel MH, Menon AG, Morreau H, Melief CJ, Offringa R, van der Burg SH, Janssen-van Rhijn C, Ensink NG, Tollenaar RA, van de Velde CJ, Kuppen PJ (2004) Dendritic cells in colorectal cancer correlate with other tumor-infiltrating immune cells. Cancer Immunol Immunother 53: 978-986.

Della Porta M, Danova M, Rigolin GM, Brugnatelli S, Rovati B, Tronconi C, Fraulini C, Russo Rossi A, Riccardi A, Castoldi G (2005) Dendritic cells and vascular endothelial growth factor in colorectal cancer: correlations with clinicobiological findings. Oncology 68: 276-284.

Edge SB, Compton CC (2010) The American Joint Committee on Cancer: the 7th edition of the AJCC cancer staging manual and the future of TNM. Ann Surg Oncol 17: 1471-1474.

Feijoo E, Alfaro C, Mazzolini G, Serra P, Penuelas I, Arina A, Huarte E, Tirapu I, Palencia B, Murillo O, Ruiz J, Sangro B, Richter JA, Prieto J, Melero I (2005) Dendritic cells delivered inside human carcinomas are sequestered by interleukin-8. Int J Cancer 116: 275-281.

Gabrilovich DI, Ostrand-Rosenberg S, Bronte V (2012) Coordinated regulation of myeloid cells by tumours. Nat Rev Immunol 12: 253-268.

Gulubova MV, Ananiev JR, Vlaykova TI, Yovchev Y, Tsoneva V, Manolova IM (2012) Role of dendritic cells in progression and clinical outcome of colon cancer. Int J Colorectal Dis 27: 159-169.

Inoue Y, Nakayama Y, Minagawa N, Katsuki T, Nagashima N, Matsumoto K, Shibao K, Tsurudome Y, Hirata K, Nagata N, Itoh H (2005) Relationship between interleukin-12-expressing cells and antigen-presenting cells in patients with colorectal cancer. Anticancer Res 25: 3541-3546.

Iwata-Kajihara T, Sumimoto H, Kawamura N, Ueda R, Takahashi T, Mizuguchi H, Miyagishi M, Takeda K, Kawakami Y (2011) Enhanced cancer immunotherapy using STAT3-depleted dendritic cells with high Th1-inducing ability and resistance to cancer cell-derived inhibitory factors. J Immunol 187: 27-36.

Kim R, Emi M, Tanabe K, Arihiro K (2006) Tumor-driven evolution of immunosuppressive networks during malignant progression. Cancer Res 66: 5527-5536.

Lin WW, Karin M (2007) A cytokine-mediated link between innate immunity, inflammation, and cancer. J Clin Invest 117: 1175-1183.

Lonnroth C, Andersson M, Arvidsson A, Nordgren S, Brevinge H, Lagerstedt K, Lundholm K (2008) Preoperative treatment with a nonsteroidal anti-inflammatory drug (NSAID) increases tumor tissue infiltration of seemingly activated immune cells in colorectal cancer. Cancer Immun 8: 5.

Michielsen AJ, Hogan AE, Marry J, Tosetto M, Cox F, Hyland JM, Sheahan KD, O’Donoghue DP, Mulcahy HE, Ryan EJ, O'Sullivan JN (2011) Tumour tissue microenvironment can inhibit dendritic cell maturation in colorectal cancer. PLoS One 6: e27944.

Michielsen AJ, Noonan S, Martin P, Tosetto M, Marry J, Biniecka M, Maguire AA, Hyland JM, Sheahan KD, O'Donoghue DP, Mulcahy HE, Fennelly D, Ryan EJ, O'Sullivan JN (2012) Inhibition of dendritic cell maturation by the tumor microenvironment correlates with the survival of colorectal cancer patients following bevacizumab treatment. Mol Cancer Ther 11: 1829-1837.

Muthuswamy R, Berk E, Junecko BF, Zeh HJ, Zureikat AH, Normolle D, Luong TM, Reinhart TA, Bartlett DL, Kalinski P (2012) NF-kappaB hyperactivation in tumor tissues allows tumor-selective reprogramming of the chemokine microenvironment to enhance the recruitment of cytolytic T effector cells. Cancer Res 72: 3735-3743.

Narita Y, Kitamura H, Wakita D, Sumida K, Masuko K, Terada S, Nakano K, Nishimura T (2013) The key role of IL-6-arginase cascade for inducing dendritic cell-dependent CD4(+) T cell dysfunction in tumor-bearing mice. J Immunol 190: 812-820.

Nefedova Y, Huang M, Kusmartsev S, Bhattacharya R, Cheng P, Salup R, Jove R, Gabrilovich D (2004) Hyperactivation of STAT3 is involved in abnormal differentiation of dendritic cells in cancer. J Immunol 172: 464-474.

Palucka K, Banchereau J (2012) Cancer immunotherapy via dendritic cells. Nat Rev Cancer 12: 265-277.

Rossowska J, Pajtasz-Piasecka E, Szyda A, Krawczenko A, Zietara N, Dus D (2009) Tumour antigen-loaded mouse dendritic cells maturing in the presence of inflammatory cytokines are potent activators of immune response in vitro but not in vivo. Oncol Rep 21: 1539-1549.

Scarlett UK, Rutkowski MR, Rauwerdink AM, Fields J, Escovar-Fadul X, Baird J, Cubillos-Ruiz JR, Jacobs AC, Gonzalez JL, Weaver J, Fiering S, Conejo-Garcia JR (2012) Ovarian cancer progression is controlled by phenotypic changes in dendritic cells. J Exp Med 209: 495-506.

Schwaab T, Weiss JE, Schned AR, Barth Jr RJ (2001) Dendritic Cell Infiltration in Colon Cancer. J Immunother (1991) 24: 130-137.

Seton-Rogers S (2012) Tumour immunology: dendritic cell switch. Nat Rev Cancer 12: 230.

Stanilov NS, Miteva LD, Dobreva ZG, Jovchev JP, Cirovski GM, Stanilova SA (2012) Monocytes expression of IL-12 related and IL-10 genes in association with development of colorectal cancer. Mol Biol Rep 39: 10895-10902.

Yuan A, Steigen SE, Goll R, Vonen B, Husbekk A, Cui G, Florholmen J (2008) Dendritic cell infiltration pattern along the colorectal adenoma-carcinoma sequence. APMIS 116: 445-456.

This work is published under the standard license to publish agreement. After 12 months the work will become freely available and the license terms will switch to a Creative Commons AttributionNonCommercial-Share Alike 3.0 Unported License. 